\title{
The Development of the Genital Psoriasis Sexual Frequency Questionnaire (GenPs-SFQ) to Assess the Impact of Genital Psoriasis on Sexual Health
}

\author{
Alice B. Gottlieb - Brian Kirby · Caitriona Ryan · April N. Naegeli · \\ Russel Burge - Alison Potts Bleakman - Milena D. Anatchkova • \\ Jennifer Cather
}

Received: September 29, 2017 / Published online: December 4, 2017

(C) The Author(s) 2017. This article is an open access publication

\section{ABSTRACT}

Introduction: Patient-reported outcome measures (PROs) exist for psoriasis but not genital psoriasis (GenPs).

Methods: This cross-sectional, qualitative study in patients with moderate-to-severe GenPs was

Enhanced content To view enhanced content for this article go to http://www.medengine.com/Redeem/ E6FCF060275165C9.

Electronic supplementary material The online version of this article (https://doi.org/10.1007/s13555017-0212-3) contains supplementary material, which is available to authorized users.

A. B. Gottlieb ( $\square)$

New York Medical College, Metropolitan Hospital,

New York, NY, USA

e-mail: gottlieba@nychhc.org

B. Kirby · C. Ryan

St Vincent's University Hospital, Dublin, Ireland

A. N. Naegeli · R. Burge · A. Potts Bleakman

Eli Lilly and Company, Indianapolis, IN, USA

R. Burge

Winkle College of Pharmacy, Pharmacy Practice and Administrative Sciences, University of Cincinnati, Cincinnati, OH, USA

M. D. Anatchkova

Evidera, Bethesda, MD, USA

J. Cather

Modern Dermatology, Aesthetics Center Dallas,

Dallas, TX, USA conducted to support development of a PRO for measuring the impact of GenPs on sexual activity and to establish content validity. The impacts of GenPs were identified in a literature review. Findings from the literature review were discussed with clinicians, and then patients with GenPs were interviewed.

Results: From the literature review, 52 articles, 44 abstracts, and 41 clinical trials met predefined search criteria. Of these, 11 concepts emerged as having theoretical support for use as measurable impacts of psoriasis symptoms on patients; these concepts included sexual functioning and general health-related quality of life (HRQoL). These concepts were confirmed and expanded upon by two clinicians who routinely care for patients with GenPs. Interviews were then conducted with GenPs patients $(n=20)$ to discuss the impact of GenPs on their HRQoL. Eighty percent of patients reported that GenPs impacted sexual frequency. The two-item GenPs Sexual Frequency Questionnaire (GenPs-SFQ) was developed to assess limitations on sexual activity frequency because of GenPs. Cognitive debriefing with an additional 50 patients with GenPs confirmed the utility and understandability of the GenPs-SFQ.

Conclusion: The GenPs-SFQ may have utility in clinical trials involving GenPs patients and in routine clinical practice.

Funding: Eli Lilly and Company. 
Plain Language Summary: Plain language summary available for this article.

Keywords: Genital psoriasis; Patient-reported outcomes; Psoriasis; Quality of life

\section{PLAIN LANGUAGE SUMMARY}

Psoriasis is a disease that causes people to have raised red patches of skin. Psoriasis can cover any part of the body, may be very itchy, and can sometimes bleed. Psoriasis currently cannot be cured, but medicines can make the raised red patches of skin smaller or completely go away. Many patients with psoriasis have raised red patches of skin on their genital area. These raised red patches of skin may itch and bleed, so many of these patients do not have sex or have sex less often than they would if they did not have raised red patches of skin on their genital area. Many patients find talking about this kind of psoriasis very difficult, even with their doctor. On the basis of research and talking with doctors and these patients, we have developed two questions for use in doctors' offices and during clinical studies that measure the effects of psoriasis on the genital area, specifically on how often these patients engage in sexual activity and how often their genital psoriasis causes them to avoid sexual activity. Patients may answer these questions before, during, and after taking medicine to treat psoriasis on their genital area. The answers to these questions may help doctors learn how well certain medicines work for psoriasis that affects the genital area.

\section{INTRODUCTION}

At some point during the course of their disease, up to $63 \%$ of patients with chronic plaque psoriasis have psoriatic lesions in the genital area [1-3]. Sexual impairment and pruritus are major issues for patients with genital psoriasis (GenPs) [3-5]. Health-related quality of life (HRQoL) is significantly worse in psoriasis patients with genital involvement compared with those without, even after correcting for overall psoriasis severity [3, 4].

GenPs has a significant impact on sexual health $[3,4,6,7]$. Women with psoriasis in the genital region have especially high levels of sexual distress [4]. Compared with patients without genital involvement, those with genital involvement experience a significantly higher impact on sexual function, sexual frequency, and fear of sexual relations [3]. Although GenPs significantly impacts HRQoL and sexual health, many health care professionals do not routinely question patients about or examine them for this condition $[8,9]$. Likewise, patients frequently do not discuss genital involvement with health care providers $[8,10,11]$.

Although GenPs has a substantial impact on patient well-being and HRQoL, the effect of therapy on psoriasis in the genital region has been tested in only a few open-label clinical trials [12-14]. Numerous patient-reported outcome measures (PROs) are available for measuring symptoms and the impact of overall psoriasis on HRQoL [15, 16], but none are specific to GenPs. However, a few PROs related to sexual health have been used to assess sexual health in a study involving patients with GenPs [4]: the Female Sexual Function Index [17, 18], the International Index of Erectile Function [19], the Female Sexual Distress Scale [18, 20], and the Sexual Quality of Life Questionnaire for use in Men [21]; in addition, patients were asked whether "they believe that since the onset of psoriasis sexual activity has declined" $[4,6]$.

The purpose of this study is to report the development and content validation of a PRO that measures the impact of GenPs on sexual health. This instrument-the Genital Psoriasis Sexual Frequency Questionnaire (GenPs-SFQ) has the potential to be a clinically meaningful standard assessment tool in routine clinical practice and in clinical trials involving patients with GenPs.

\section{METHODS}

\section{Study Objectives and Design}

This was a cross-sectional study designed to support development and content validity of a new PRO in GenPs patients. A five-step study design was employed: (a) literature review to document the impact of GenPs on sexual 
activity and to identify concepts of GenPs, (b) clinician input to confirm the concepts of GenPs that are of greatest clinical relevance to patients, (c) concept elicitation interviews with GenPs patients, (d) item generation, and (e) cognitive debriefing evaluation.

\section{Literature Review}

A targeted literature search was conducted to identify peer-reviewed articles and conference proceedings that discussed GenPs and its impact on HRQoL and sexual health. English-language articles published between 2005 and 2015 or conference abstracts published between 2011 and 2015 and indexed in EMBASE/Medline were included (see Table S1 in the supplementary material for search terms). A separate search of www.clinicaltrials.gov was performed to identify PROs used in future or active clinical trials between 2013 and 2015.

\section{Clinician Input}

After completion of the literature review, two USA-based dermatologists were interviewed including one author of this manuscript (CR); these clinicians had extensive experience in treating GenPs and experience in clinical trials involving psoriasis patients. The interviews' purpose was to confirm the GenPs impacts most relevant to patients from the clinician's perspective.

\section{Concept Elucidation}

Participants were recruited in the USA from five clinical sites located in Arkansas, Indiana, Michigan, and Washington. Participants were eligible if, at screening, they were aged at least 18 years and had confirmed chronic plaque psoriasis for at least 6 months, had an affected body surface area of at least $1 \%$, and had a current or recent history (within 3 months) of moderate or severe genital involvement (Patient Global Assessment score $\geq 4$ on a 6 -point scale from 0 to 5) as reported by the patient and confirmed by the site investigator. Participants were eligible if they failed to respond to or had been intolerant of one or more topical therapies for GenPs.

One-on-one participant interviews were planned as in-person or telephone interviews using a semistructured guide. Interviews were audio-recorded and transcribed, and the transcripts were de-identified. Interviews were conducted between August 19, 2015, and November 19, 2015. All procedures followed were in accordance with the ethical standards of the responsible committee on human experimentation (institutional and national) and with the Helsinki Declaration of 1964, as revised in 2013. Written and verbal informed consent for audio recording was obtained from all participants for being included in the study, although the study was determined to be "exempt" by the local institutional review board (Chesapeake IRB, Columbia, MD, USA). Before the telephone interviews were conducted, packets containing the informed consent form and study materials were mailed to participants.

Concept elicitation was performed during the interviews. Participants were asked open-ended questions without any definitions of symptoms or impacts and were then questioned on predefined symptoms and impacts. Each participant's interview was completed in one session lasting approximately $2 \mathrm{~h}$. Upon completion, participants were remunerated for their time.

\section{Item Generation}

The findings of the literature review, clinician review, and concept elicitation identified and confirmed that decrements in sexual function were an important impact of GenPs. This strongly supported the development of a new PRO instrument, the GenPs-SFQ, to measure the impact of GenPs on sexual frequency for use in trials evaluating treatments for GenPs. Two global items were generated on the basis of how patients described their experience: the first addressed the frequency of sexual activity over the previous week, and the second addressed how often GenPs limited the frequency of sexual activity during the same week. 
Response options for each item were designed so that the full range of frequency was captured, reflecting what had been learned through concept elicitation; in practice, responses can be captured in the format of a weekly diary. The recall period of 1 week and weekly diary approach were selected to best capture the frequency of sexual activity and the patient's ability to recall it accurately as guided by results of the concept elicitation interviews.

\section{Cognitive Debriefing}

Cognitive debriefing interviews to confirm item comprehension and appropriateness of the final GenPs-SFQ were conducted with a second set of 50 participants from seven countries and one US territory (Australia, Austria, Belgium, Canada, Netherlands, Puerto Rico, USA, and Turkey). The newly developed instrument was tested on these adults with self-reported GenPs to ensure that each item of the GenPs-SFQ adequately captured the concepts as intended in English for the non-English-speaking countries and to confirm item comprehension and cultural appropriateness. Before the interviews, participants were asked to review the GenPsSFQ and circle any words, phrases, or sentences that were difficult to understand. During the interviews, participants were asked to identify any words or phrases they had previously circled. The interviewers then guided subjects through the documents, line-by-line, asking them to paraphrase each item. If a subject expressed difficulty understanding an item or concept, the interviewers probed to determine the reason and then elicited suggestions for rewording the difficult text.

\section{Data Analysis}

Qualitative data were based on interview notes, recordings, and transcripts and were analyzed using a content analysis approach. Participant interview transcripts during the concept elicitation phase were analyzed using ATLAS.ti version 7.5.9 (Scientific Software Development $\mathrm{GmbH}$, Berlin, Germany), which systematically identified themes within qualitative data. A coding dictionary based on the semistructured interview guide was developed. Results from the debriefing were qualitatively analyzed to determine if changes were necessary to improve the comprehensibility and relevancy of each GenPsSFQ item.

\section{RESULTS}

\section{Literature Review and Clinician Input}

Fifty-two articles, 44 abstracts, and 41 clinical trials met predefined search criteria.

From the literature search, 11 concepts emerged as having theoretical support for use as measureable impacts of psoriasis symptoms on HRQoL or sexual function (Table 1). The most frequent concepts overall were psychological feelings $(n=42,80.8 \%)$, social relationships $(n=37,71.2 \%)$, physical activities or general physical functioning $(n=31,59.6 \%)$, daily living and activities $(n=29,55.8 \%)$, general HRQoL $(n=29,55.8 \%)$, and work or school impact ( $n=26,50.0 \%)$. Sexual functioning was discussed by $19(36.5 \%)$ reviewed articles; of these, $3(5.8 \%)$ articles discussed this concept in relation to GenPs, making it the most frequently discussed concept specific to GenPs. A further targeted search of sexual function concepts was conducted by reviewing relevant conference abstracts (Table 2). Results revealed the following most frequently emergent concepts: dyspareunia ( $n=2,4.5 \%)$, increased discomfort or worsening of symptoms after sex $(n=2,4.5 \%)$, and sexual dysfunction $(n=2$, $4.5 \%)$.

During the clinician interviews, sexual function, which encompasses sexual activity, was identified as the most relevant functional impact of GenPs. One clinician emphasized the difference between sexual activity and sexual dysfunction (i.e., erectile dysfunction), indicating that GenPs impacts sexual activity, not sexual dysfunction. Specific impacts on sexual activity included fear of and avoidance of sex, pain during sexual intercourse (dyspareunia), worsening of psoriasis after intercourse, and bleeding and cracking in the genital area. 
Table 1 Summary of health-related quality of life and/or functional impact concept emergence: published articles $(n=52)$

\begin{tabular}{lllll}
\hline HRQoL/functional concepts & \multicolumn{2}{c}{ Frequency of mention, $\boldsymbol{n}$ (\%) } & \\
\cline { 2 - 5 } & Total & $\begin{array}{l}\text { In relation to } \\
\text { psoriasis }^{\mathbf{2}}\end{array}$ & $\begin{array}{l}\text { In relation to } \\
\text { genital psoriasis }\end{array}$ & $\begin{array}{l}\text { In relation to both } \\
\text { psoriasis and genital } \\
\text { psoriasis }\end{array}$ \\
\hline $\begin{array}{l}\text { Psychological feelings (e.g., mood } \\
\text { disturbance) }\end{array}$ & $42(80.8)$ & $41(78.8)$ & $1(1.9)$ & $0(0)$ \\
$\begin{array}{l}\text { Social relationships (including social } \\
\text { stigmatization) }\end{array}$ & $37(71.2)$ & $35(67.3)$ & $2(3.8)$ & $0(0)$ \\
$\begin{array}{l}\text { Physical activities or general physical } \\
\text { functioning }\end{array}$ & $31(59.6)$ & $30(57.7)$ & $1(1.9)$ & $0(0)$ \\
$\begin{array}{l}\text { Daily living and activities } \\
\text { HRQoL }\end{array}$ & $29(55.8)$ & $28(53.8)$ & $1(1.9)$ & $0(0)$ \\
Work or school & $29(55.8)$ & $28(53.8)$ & $1(1.9)$ & $0(0)$ \\
Recreation & $26(50.0)$ & $25(48.1)$ & $1(1.9)$ & $0(0)$ \\
Sexual functioning & $20(38.5)$ & $19(36.5)$ & $1(1.9)$ & $0(0)$ \\
Vitality & $19(36.5)$ & $15(28.8)$ & $3(5.8)$ & $1(1.9)$ \\
Financial & $7(13.5)$ & $7(13.5)$ & $0(0)$ & $0(0)$ \\
Cognitive functioning & $4(7.7)$ & $4(7.7)$ & $0(0)$ & $0(0)$ \\
\hline
\end{tabular}

Fifty-two articles met the inclusion criteria, but only a subset explicitly discussed health-related quality of life and/or functional impact concept emergence

HRQOL Health-related quality of life

a Articles that discussed symptoms in the context of psoriasis in general

b Articles that discussed symptoms specifically in the context of genital psoriasis

c Articles that discussed symptoms in the context of both psoriasis in general and specifically for genital psoriasis

Avoidance of sex was noted by one clinician as more commonly mentioned by female patients as opposed to male patients. Psychological impacts of GenPs, such as depression and anxiety, were also mentioned by one clinician as affecting patients. One clinician noted the impact of sexual activity impairment on the patient's partner. A clinician recommended developing an instrument to assess the impact of GenPs on sexual health and HRQoL.

\section{Concept Elicitation Interviews}

Twenty-two of 25 screened participants met the study criteria. Two eligible participants were unavailable; therefore, 20 participants were interviewed. The mean age of participants was $45 \pm 14.2$ years (range $21-68$ ); $45 \%$ were male, and $90 \%$ were white (Table 3 ). All participants chose telephone interviews rather than in-person interviews.

At the beginning of the interview, GenPs was defined as psoriasis occurring on the "outer lip" (labia majora), "inner lip" (labia minora), and "area between the vagina and the anus" (perineum) for females and on the penis, scrotum, and "area between the penis and the anus" (perineum) for males. During the patient interviews, sexual functioning was the most common spontaneously endorsed impact of GenPs, 
Table 2 Summary of sexual function concepts: conference proceedings $(n=44)$

\begin{tabular}{lllll}
\hline Sexual function concepts & \multicolumn{4}{l}{ Frequency of mention, $\boldsymbol{n}(\%)$} \\
\cline { 2 - 5 } & Total & $\begin{array}{l}\text { In relation to } \\
\text { psoriasis }^{\mathbf{a}}\end{array}$ & $\begin{array}{l}\text { In relation to } \\
\text { genital psoriasis }^{\mathbf{b}}\end{array}$ & $\begin{array}{l}\text { In relation to both psoriasis and } \\
\text { genital psoriasis }^{\mathbf{c}}\end{array}$ \\
\hline $\begin{array}{l}\text { Dyspareunia } \\
\begin{array}{l}\text { Increased discomfort/worsening of } \\
\text { symptoms after sex }\end{array}\end{array}$ & $2(4.5)$ & $1(2.3)$ & $1(2.3)$ & $0(0)$ \\
Sexual dysfunction & $2(4.5)$ & $1(2.3)$ & $0(0)$ & $0(0)$ \\
Bleeding during sex & $1(2.3)$ & $1(2.3)$ & $0(0)$ & $1(2.3)$ \\
Difficulty moving during sex & $1(2.3)$ & $1(2.3)$ & $0(0)$ & $0(0)$ \\
Ripping of skin during sex & $1(2.3)$ & $1(2.3)$ & $0(0)$ & $0(0)$ \\
Vaginal dryness & $1(2.3)$ & $1(2.3)$ & $0(0)$ & $0(0)$ \\
\hline
\end{tabular}

Forty-four conference proceedings met the inclusion criteria, but only a subset explicitly discussed sexual function concepts

${ }^{a}$ Conference proceedings that discussed symptoms in the context of psoriasis in general

b Conference proceedings that discussed symptoms specifically in the context of genital psoriasis

c Conference proceedings that discussed symptoms in the context of both psoriasis in general and specifically for genital psoriasis

with $18(90 \%)$ participants spontaneously endorsing one or more aspects of sexual impact. When asked about impact of GenPs on sexual activity (defined as not limited to intercourse and including activities such as masturbation), the most common impacts of GenPs were on sexual frequency, sexual experience, and symptom worsening after sexual activity, each reported by $80 \%$ of participants. Table 4 shows other impacts of GenPs on sexual activity. Upon reviewing the transcripts of the patient interviews, reduced sexual frequency and avoidance of sexual activity and/or intimate relationships because of physical symptoms or embarrassment were common themes. Table 5 shows representative participant responses. Because of the non-complex concepts of interest, saturation was achieved with the 20 participants (data not shown).

\section{Development of GenPs-SFQ}

On the basis of the key experiences from the participant interviews, avoidance of sexual activity with a subsequent impact on frequency of sexual activity was a key theme. The two-item GenPs-SFQ was created to add frequency to the concept of avoidance of sexual activity (Table 6). The GenPs-SFQ provides quantification of the impact of GenPs on frequency and limitations of sexual activity and focuses specifically on the impact of GenPs on sexual frequency/activity on the basis of the patient's response to item 1 ("In the past week, how many times did you engage in sexual activity?"). This in combination with item 2 ("In the past week, how often did your genital psoriasis limit the frequency of your sexual activity?") distinguishes between patients who avoid sexual activity because of GenPs versus reasons unrelated to GenPs. The GenPs-SFQ defines the genital area as the labia majora (outer lips), labia minora (inner lips), and perineum (area between vagina and anus) for females and the penis, scrotum, and perineum (area between the penis and anus) for males. The recall period is 1 week. 
Table 3 Patient demographics

\begin{tabular}{|c|c|c|}
\hline Characteristics & $\begin{array}{l}\text { Number of patients } \\
\text { responding }\end{array}$ & $n(\%)$ or mean $(\mathrm{SD})$ \\
\hline Age, years [mean $(\mathrm{SD})]$ & 20 & $45(14.2)$ \\
\hline Sex, $n(\%)$ & 20 & \\
\hline Male & - & $9(45)$ \\
\hline Female & - & $11(55)$ \\
\hline Race, $n(\%)$ & 20 & \\
\hline White & - & $18(90)$ \\
\hline Black or African-American & - & $1(5)$ \\
\hline Two or more races & - & $1(5)$ \\
\hline Duration of psoriasis, years [mean $(\mathrm{SD})]$ & 19 & $18(14)$ \\
\hline Duration of genital psoriasis, years [mean (SD)] & 20 & $7.5(9.7)$ \\
\hline BSA score, mean (SD) & 15 & $10.4(12.7)$ \\
\hline Self-reported general health within past week, $n$ (\%) & 20 & - \\
\hline Excellent & - & $2(10)$ \\
\hline Very good & - & $4(20)$ \\
\hline Good & - & $11(55)$ \\
\hline Fair & - & $3(15)$ \\
\hline Poor & - & 0 \\
\hline Self-reported severity of overall psoriasis symptoms (worst over past 3 months), $n$ (\%) & 20 & - \\
\hline 0 (clear) & - & 0 \\
\hline 1 & - & $1(5)$ \\
\hline 2 & - & $1(5)$ \\
\hline 3 & - & $5(25)$ \\
\hline 4 & - & $5(25)$ \\
\hline 5 (severe) & - & $8(40)$ \\
\hline $\begin{array}{l}\text { Self-reported severity of genital psoriasis symptoms (worst over past } 3 \text { months), } n \\
(\%)^{\mathrm{a}}\end{array}$ & 20 & - \\
\hline 0 (clear) & - & 0 \\
\hline 1 & - & 0 \\
\hline 2 & & $1(5)$ \\
\hline 3 & - & $5(25)$ \\
\hline 4 & & $8(40)$ \\
\hline 5 (severe) & - & $6(30)$ \\
\hline Sexual activity status, $n(\%)$ & 20 & - \\
\hline Not active & - & $9(45)$ \\
\hline Active & - & $9(45)$ \\
\hline Not asked ${ }^{\mathrm{b}}$ & - & $2(10)$ \\
\hline Currently receiving treatment for overall psoriasis, $n$ (\%) & 20 & $14(70)$ \\
\hline
\end{tabular}

$B S A$ body surface area

${ }^{a}$ All participants met eligibility criteria (Patient Global Assessment $\geq 4$, 6-point scale from 0 to 5) at time of screening; the table reflects responses at the time of the interview

$\mathrm{b}$ The question was not asked because of conversation flow, auditory cues, and subject's apparent lack of comfort with sensitive topics per interviewer judgment 
Table 4 Functional impact concepts emerging from patient interviews

\begin{tabular}{llcl}
\hline Sexual impact concepts & \multicolumn{4}{l}{ Frequency of mention, $\boldsymbol{n}(\%)$} \\
\cline { 2 - 4 } & Total & Spontaneous & After probing \\
\hline Decreased sexual frequency & $16(80)$ & $7(35)$ & $9(45)$ \\
Worsening of genital psoriasis symptoms after sexual activity & $16(80)$ & $12(60)$ & $4(20)$ \\
Negative effects on sexual experience & $16(80)$ & $13(65)$ & $3(15)$ \\
Avoidance of sexual relationships & $15(75)$ & $12(60)$ & $3(15)$ \\
Reduced sexual desire & $11(55)$ & $2(10)$ & $9(45)$ \\
\hline
\end{tabular}

$n$ number of patients

Table 5 Patient-reported impacts of genital psoriasis on sexual frequency and avoidance

\section{Representative patient quotations}

Decreased sexual frequency

It's decreased it a lot

I haven't had sex in about 5 months

Well, you know, you, there's no sex life

I'm not as sexually active now as when I was, you know, 25 or 21, but so it, it's, it has gotten to the point you just don't have relations

I would say it's easily cut my frequency down in half

It's, it's taken it away just because, I mean, it's just too painful

No, I, it doesn't, unless, unless there's an injury. That's the only time I'm kind of apprehensive about it

Yeah, so I don't do it much.... Not very often. It really affects that, trust me

\section{Avoidance of sexual relationships}

Well, you know, it's just another factor that you have in your mind that you really wouldn't want to expose that to anyone or have to explain it or have them question you. So you'd rather just skip the whole thing... It probably weighs on the side of I'm not looking for anybody

No, not currently right now because it kind of comes and goes in stages. I have been off and on but there's definitely a lot of stress because you don't know if it's going to flare up that week or next week or when and it's just a lot to deal with. There's not many girls that would be too understanding of what's going on. Most people think it's cracking and dry skin and not a lot of people understand psoriasis so they would think, you know, STD or this or that or what's wrong with you or something. So it's just I've steered away from it, I guess, a little bit

Semistructured interviews were conducted with 20 patients. Some patient quotes were edited to minimize redundant language 
Table 6 Genital psoriasis sexual frequency questionnaire (GenPs-SFQ)

\begin{tabular}{|c|c|}
\hline $\begin{array}{l}\text { Please answer the questions based on your psoriasis symptoms in the genital area }{ }^{a} \text { within the past } \\
\text { week }\end{array}$ & $\begin{array}{l}\text { Response } \\
\text { options }\end{array}$ \\
\hline \multirow[t]{3}{*}{ In the past week, how many times did you engage in sexual activity ${ }^{b}$ ? } & None/zero \\
\hline & Once \\
\hline & Two or more \\
\hline \multirow[t]{5}{*}{ In the past week, how often did your genital psoriasis limit the frequency of your sexual activity ${ }^{b}$ ? } & Always \\
\hline & Often \\
\hline & Sometimes \\
\hline & Rarely \\
\hline & Never \\
\hline
\end{tabular}

For permission to reproduce or use the GenPs-SFQ, please contact copyright@lilly.com

a Genital area is defined as the labia majora (outer lips), labia minora (inner lips), and perineum (area between vagina and anus) for females and the penis, scrotum, and perineum (area between the penis and anus) for males

b Not limited to sexual intercourse and includes activities such as masturbation

\section{Cognitive Debriefing}

Cognitive debriefing was conducted with 50 participants with an average age of $47.5 \pm 14.6$ years (range 18-82) and an average of $13 \pm 3.8$ years of academic education (range 6-23 years). Forty-four percent of participants were male. Patients did not report difficulties interpreting or using the GenPs-SFQ items, so no changes were made after the cognitive debriefing. Overall, the cognitive interviews revealed that the instructions and items of the GenPs-SFQ adequately capture the concepts as intended and were, overall, easily understood in English and other languages and confirmed as culturally appropriate by patients with GenPs residing in the seven countries and one US territory that will be included in the planned clinical trials.

No participant indicated difficulty in making a rating using the frequency rating scales. Participants used lower ratings on the scale to indicate less frequent sexual activity for item 1 and/or less limitation of sexual activity because of GenPs for item 2. Higher ratings were used to describe more frequent sexual activity and/or more limitation in sexual activity because of GenPs, respectively. Participants thought the recall period of 1 week was appropriate, made sense, and was relevant. Participants were able to make ratings based strictly on this recall period and reported no difficulty in remembering their experiences over that period.

\section{DISCUSSION}

Although multiple PROs exist for measuring the impact of general psoriasis on HRQoL $[15,16]$ and for measuring sexual function [22], none had been developed or validated specifically for use with GenPs. Using the concept of sexual activity avoidance, the GenPs-SFQ was developed specifically for individuals with GenPs. However, the broader concept of sexual activity impacts in other psoriasis populations has been reported. In a survey of 481 Dutch patients with psoriasis, including patients with genital lesions at the time of the survey, $24.9 \%(32.8 \%$ of females; $19.1 \%$ of males) reported that sexual activity declined after the onset of psoriasis [4]. 
The GenPs-SFQ measures the impact of GenPs on patients' lives in terms of frequency of sexual activity. Although the GenPs was developed de novo, item 2 ("In the past week, how often did your genital psoriasis limit the frequency of your sexual activity?") does have some similarity to item 9 of the Dermatology Life Quality Index ("Over the last week, how much has your skin caused any sexual difficulties?") [23]. These items are easy to understand, and the GenPs-SFQ can be administered to patients either on paper or electronically. The scale is specific to GenPs and is potentially useful in both clinical trials and routine clinical practice. The work described here establishes content validity, which is an initial step in the development of a new PRO [24-26]. The development of the initial items and participant interviews were conducted in accordance with guidelines and best practices for validating content [24-26].

There may be limitations in the extent to which these findings can be generalized to all GenPs patients. The sample size $(N=20)$ appears small, but saturation, which is the point whereby gathering additional data does not yield new concepts [27], was reached with 20 patients; saturation is the recommended method for determining the sample size for concept elicitation interviews [26]. Racial minorities were underrepresented in the original sample of 20 patients. Sexual orientation was not included in the baseline demographic questions. Notably, all patients chose telephone interviews over in-person interviews, and this should be noted for future studies; it is possible that patients may have been less forthcoming if in-person interviews had been conducted. Finally, the described work is qualitative; therefore, psychometric properties of the GenPs-SFQ still need to be evaluated to determine the instrument's reliability, validity, and ability to measure changes in patient wellbeing.

\section{CONCLUSIONS}

The GenPs-SFQ may help obtain information that is relevant to understanding the burden of genital psoriasis and inform potential treatment in both clinical and research settings. Practitioners should consider ways to make patients feel more comfortable discussing their GenPs.

\section{ACKNOWLEDGEMENTS}

Funding. This study was funded by Eli Lilly and Company, Indianapolis, Indiana, USA which contracted with Evidera (Bethesda, Maryland) for the design and analysis of the study. Article processing charges were funded by Eli Lilly and Company, Indianapolis, IN, USA. All authors had full access to all of the data in this study and take complete responsibility for the integrity of the data and accuracy of the data analysis.

Authorship. All named authors meet the International Committee of Medical Journal Editors (ICMJE) criteria for authorship for this manuscript, take responsibility for the integrity of the work as a whole, and have given final approval to the version to be published.

Medical Writing and/or Editorial Assistance. Writing and editorial assistance in the preparation of this manuscript were provided by Lori Kornberg, PhD (INC Research, Raleigh, NC), Meredith Fraser, MFA (INC Research, Raleigh, NC), and Kristin Hollister, PhD (Eli Lilly and Company, Indianapolis, IN). Support for this assistance was funded by Eli Lilly and Company. The authors would like to thank the participants who shared the personal details of their genital psoriasis.

Disclosures. A. Gottlieb is a consultant and advisory board member for Janssen, Celgene, Bristol Myers Squibb, Beiersdorf, AbbVie, UCB, Novartis, Incyte, Eli Lilly and Company, Dr Reddy's Laboratories, Valeant, Dermira, Allergan, and Sun Paharmaceutical Industries. She received research and educational grants from Janssen and Incyte. 
B. Kirby receives research grants from AbbVie, Novartis, Merck-Sharpe-Dolme, and Pfizer. He has acted as a consultant and/or speaker for AbbVie, Novartis, Janssen, Celgene, Almirall, Eli Lilly and Company, and LEO Pharma.

C. Ryan has acted as an advisor and/or speaker for AbbVie, Aqua, Dermira, Dr Reddy's Laboratories, Eli Lilly and Company, Janssen, Medimetriks, Novartis, Regeneron-Sanofi, UCB, and XenoPort.

M. Anatchkova is an employee of Evidera. Evidera received research study support from Eli Lilly and Company.

J. Cather is in the speaker bureaus of AbbVie, Celgene, Eli Lilly and Company, and Janssen; received honoraria from AbbVie, Eli Lilly and Company, Janssen, and Novartis; is a consultant for AbbVie, Actelion, Eli Lilly and Company, and Janssen; is an investigator for Celgene, Cutanea, Dermira, GaldermaLabs, GlaxoSmithKline, Janssen, Merck, Novartis, Pfizer, Regeneron, Sandoz, TolmarPharma, Vitae, and XenoPort; has received grants from Allergan, Celgene, Cutanea, Dermira, GlaxoSmithKline, GaldermaLabs, Janssen, MSD, Novartis, Pfizer, Regeneron, Sandoz, TolmarPharma, Vitae, and XenoPort; and has received other financial benefit from AbbVie.

A. N. Naegeli is a full-time employee of Eli Lilly and Company and owns stock.

A. Potts Bleakman is a full-time employee of Eli Lilly and Company and owns stock.

R. Burge is a full-time employee of Eli Lilly and Company and owns stock.

Compliance with Ethics Guidelines. All procedures followed were in accordance with the ethical standards of the responsible committee on human experimentation (institutional and national) and with the Helsinki Declaration of 1964 , as revised in 2013. Written and verbal informed consent for audio recording was obtained from all participants for being included in the study, although the study was determined to be "exempt" by the local IRB (Chesapeake IRB, Columbia, MD).

Data Availability. The datasets generated during and/or analyzed during the current study are in the form of audio recordings and transcripts, and are not publicly available because of patient privacy.

Open Access. This article is distributed under the terms of the Creative Commons Attribution-NonCommercial 4.0 International License (http://creativecommons.org/licenses/ by-nc/4.0/), which permits any noncommercial use, distribution, and reproduction in any medium, provided you give appropriate credit to the original author(s) and the source, provide a link to the Creative Commons license, and indicate if changes were made.

\section{REFERENCES}

1. Fouéré S, Adjadj L, Pawin H. How patients experience psoriasis: results from a European survey. J Eur Acad Dermatol Venereol. 2005;19(Suppl 3):2-6.

2. Meeuwis KA, de Hullu JA, Massuger LF, van de Kerkhof PC, van Rossum MM. Genital psoriasis: a systematic literature review on this hidden skin disease. Acta Derm Venereol. 2011;91(1):5-11.

3. Ryan C, Sadlier M, De Vol E, et al. Genital psoriasis is associated with significant impairment in quality of life and sexual functioning. J Am Acad Dermatol. 2015;72(6):978-83.

4. Meeuwis KA, de Hullu JA, van de Nieuwenhof HP, et al. Quality of life and sexual health in patients with genital psoriasis. Br J Dermatol. 2011;164(6):1247-55.

5. Zamirska A, Reich A, Berny-Moreno J, Salomon J, Szepietowski JC. Vulvar pruritus and burning sensation in women with psoriasis. Acta Derm Venereol. 2008;88(2):132-5.

6. Gupta MA, Gupta AK. Psoriasis and sex: a study of moderately to severely affected patients. Int J Dermatol. 1997;36(4):259-62.

7. Meeuwis KA, de Hullu JA, IntHout J, et al. Genital psoriasis awareness program: physical and psychological care for patients with genital psoriasis. Acta Derm Venereol. 2015;95(2):211-6.

8. Menter A, Korman NJ, Elmets CA, et al. Guidelines of care for the management of psoriasis and psoriatic arthritis: section 6 . Guidelines of care for the treatment of psoriasis and psoriatic arthritis: casebased presentations and evidence-based conclusions. J Am Acad Dermatol. 2011;65(1):137-74. 
9. Farber EM, Nall L. Genital psoriasis. Cutis. 1992;50(4):263-6.

10. Andreassi L, Bilenchi R. Non-infectious inflammatory genital lesions. Clin Dermatol. 2014;32(2): 307-14.

11. Meeuwis KA, van de Kerkhof PC, Massuger LF, de Hullu JA, van Rossum MM. Patients' experience of psoriasis in the genital area. Dermatology. 2012;224(3):271-6.

12. Bissonnette R, Nigen S, Bolduc C. Efficacy and tolerability of topical tacrolimus ointment for the treatment of male genital psoriasis. J Cutan Med Surg. 2008;12(5):230-4.

13. Jemec GB, Baadsgaard O. Effect of cyclosporine on genital psoriasis and lichen planus. J Am Acad Dermatol. 1993;29(6):1048-9.

14. Rallis E, Nasiopoulou A, Kouskoukis C, et al. Successful treatment of genital and facial psoriasis with tacrolimus ointment $0.1 \%$. Drugs Exp Clin Res. 2005;31(4):141-5.

15. Feldman SR, Krueger GG. Psoriasis assessment tools in clinical trials. Ann Rheum Dis. 2005;64(2):ii65-8 (discussion ii69-73).

16. Kitchen H, Cordingley L, Young H, Griffiths CE, Bundy C. Patient-reported outcome measures in psoriasis: the good, the bad and the missing! $\mathrm{Br} \mathrm{J}$ Dermatol. 2015;172(5):1210-21.

17. Rosen R, Brown C, Heiman J, et al. The Female Sexual Function Index (FSFI): a multidimensional self-report instrument for the assessment of female sexual function. J Sex Marital Ther. 2000;26(2):191-208.

18. ter Kuile MM, Brauer M, Laan E. The Female Sexual Function Index (FSFI) and the Female Sexual Distress Scale (FSDS): psychometric properties within a Dutch population. J Sex Marital Ther. 2006;32(4): 289-304.

19. Rosen RC, Riley A, Wagner G, Osterloh IH, Kirkpatrick J, Mishra A. The International Index of Erectile Function (IIEF): a multidimensional scale for assessment of erectile dysfunction. Urology. 1997;49(6):822-30.
20. Derogatis LR, Rosen R, Leiblum S, Burnett A, Heiman J. The Female Sexual Distress Scale (FSDS): initial validation of a standardized scale for assessment of sexually related personal distress in women. J Sex Marital Ther. 2002;28(4):317-30.

21. Abraham L, Symonds T, Morris MF. Psychometric validation of a sexual quality of life questionnaire for use in men with premature ejaculation or erectile dysfunction. J Sex Med. 2008;5(3):595-601.

22. DeRogatis LR. Assessment of sexual function/dysfunction via patient reported outcomes. Int J Impot Res. 2008;20(1):35-44.

23. Finlay AY, Khan GK. Dermatology Life Quality Index (DLQI) — a simple practical measure for routine clinical use. Clin Exp Dermatol. 1994;19(3): 210-6.

24. US Department of Health and Human Services. Food and Drug Administration. Guidance for Industry: patient-reported outcome measures: use in medical product development to support labeling claims. December 2009. http://www.fda.gov/ downloads/Drugs/Guidances/UCM193282.pdf. Accessed 28 Sep 2016.

25. Patrick DL, Burke LB, Gwaltney CJ, et al. Content validity-establishing and reporting the evidence in newly developed patient-reported outcomes (PRO) instruments for medical product evaluation: ISPOR PRO Good Research Practices Task Force report: part 2-assessing respondent understanding. Value Health. 2011;14(8):978-88.

26. Patrick DL, Burke LB, Gwaltney CJ, et al. Content validity-establishing and reporting the evidence in newly developed patient-reported outcomes (PRO) instruments for medical product evaluation: ISPOR PRO Good Research Practices Task Force report: part 1-eliciting concepts for a new PRO instrument. Value Health. 2011;14(8):967-77.

27. Rothman M, Burke L, Erickson P, Leidy NK, Patrick DL, Petrie CD. Use of existing patient-reported outcome (PRO) instruments and their modification: the ISPOR Good Research Practices for Evaluating and Documenting Content Validity for the Use of Existing Instruments and Their Modification PRO Task Force report. Value Health. 2009;12(8):1075-83. 